\title{
A PRIMARY DECOMPOSITION FOR TORSION MODULES
}

\section{J. S. ALIN}

\begin{abstract}
A definition of primary module is given and a theorem is proved characterizing rings for which each torsion module, in the sense of S. E. Dickson, decomposes as a direct sum of its primary submodules. This theorem is applied to obtain a generalization of Fuchs' theorem on the additive group structure of Artinian rings.
\end{abstract}

1. Introduction. S. E. Dickson [2], [3] has investigated a primary decomposition for torsion modules over an arbitrary ring and Alin [1] has characterized rings for which this primary decomposition holds. The purpose of this note is to define "primary module" in such a way that the primary decomposition holds for a larger class of rings, in particular, for all Noetherian rings.

All rings $R$ have a unit and modules are unitary left $R$-modules. If $M$ is an $R$-module, $M^{+}$denotes the underlying additive group of $M$. It is well known that if $S$ is a simple $R$-module, then $S^{+}$is a direct sum of copies of $Z_{p}$, the cyclic group of order $p$, or a direct sum of copies of $Q$, the additive group of rational numbers. In the first case we say that $S$ is of type $p$ and in the second, $S$ is of type $Q$.

Let $p_{1}, p_{2}, \cdots$ be an indexing of the positive primes and for each $i=1,2, \cdots$ let $S_{i}$ be a representative set of simple $R$-modules of type $p_{i}$. Let $S_{0}$ be a representative set of simple $R$-modules of type $Q$. For $i=0,1, \cdots$ let $J_{i}$ be the torsion class generated by $S_{i}$ and let $J$ be the torsion class generated by $\bigcup_{i=0}^{\infty} S_{i}[4]$. Thus $J_{i}$ (respectively $J$ ) is the class of all modules $M$ such that each nonzero homomorphic image of $M$ has a submodule isomorphic to a member of $S_{i}$ (respectively, $\left.\bigcup_{i=0}^{\infty} \delta_{i}\right)$. The classes $\mathfrak{J}, \mathfrak{J}_{0}, J_{1}, \cdots$ are closed under submodules, direct sums, extensions and homomorphic images. It follows that each module $M$ has a unique largest submodule $M_{i}$ in $\mathfrak{J}_{i}$. If $M \in J_{i}, i \geqq 1, M$ is $p_{i}$-primary and if $M \in J_{0}, M$ is $Q$-primary. The modules $M \in J$ are called torsion. The primary decomposition holds for a ring $R$ if and only if for each $M \in J, M=\sum_{i=0}^{\infty} M_{i}$ (direct), i.e., each torsion module is a direct sum of its primary submodules.

For an $R$-module $M$, Soc $(M)$ denotes the socle of $M$. We let $T^{1}(M)$ $=\operatorname{Soc}(M)$ and extend to an ascending chain of submodules $\left\{T^{\alpha}(M)\right\}$

Received by the editors November 11, 1969.

AMS 1969 subject classifications. Primary 1640; Secondary 1690.

Key words and phrases. Torsion classes, Primary decomposition. 
of $M$ in the usual manner [1]. If $M \in J, T^{\alpha}(M)=M$ for some ordinal $\alpha$ and the least such ordinal is the $T$-length of $M$.

For any module $M, M_{t}$ denotes the usual torsion subgroup of the group $M^{+}$and $M_{p}$, for a prime $p$, denotes the maximum $p$-primary subgroup of $M^{+}$. Note that $M_{t}$ and $M_{p}$ are submodules of $M$.

We use $\operatorname{Ext}(A, B)$ for $\operatorname{Ext}_{R}^{1}(A, B)$ and $\operatorname{Hom}(A, B)$ for $\operatorname{Hom}_{R}(A, B)$. The reader is referred to MacLane [6] for the properties of Ext which are used in what follows.

2. The main theorem. The following lemma, in part characterizing the primary submodules of a module $M$, will be needed in the proof of the main theorem.

Lemma 2.1. Let $M \in J$. Then

(1) $M_{i}=M_{p_{i}}$ for $i \geqq 1$.

(2) $\left(M_{0}\right)+i$ is a torsion-free divisible group.

Proof. (1) Clearly $\operatorname{Soc}\left(M_{i}\right) \subseteq M_{p_{i}}$ and by induction it is easy to see that $M_{i} \subseteq M_{p_{i}}$. But $M_{i}$ and $M_{p_{i}}$ are submodules of $M$ so $M_{p_{i}} / M_{i}$ is either zero or it has a simple submodule, since $M \in J$. The latter choice leads to a contradiction, hence $M_{i}=M_{p_{i}}$.

(2) It is clear that $\operatorname{Soc}\left(M_{0}\right)$ is divisible and torsion-free. Assume inductively that $T^{\alpha}\left(M_{0}\right)$ is divisible and torsion-free. Then since

$$
T^{\alpha+1}\left(M_{0}\right) / T^{\alpha}\left(M_{0}\right)=\operatorname{Soc}\left(M_{0} / T^{\alpha}\left(M_{0}\right)\right)
$$

is a direct sum of $Q$-type simples and since

$$
0 \rightarrow T^{\alpha}\left(M_{0}\right) \rightarrow T^{\alpha+1}\left(M_{0}\right) \rightarrow T^{\alpha+1}\left(M_{0}\right) / T^{\alpha}\left(M_{0}\right) \rightarrow 0
$$

is exact, we get that $T^{\alpha+1}\left(M_{0}\right)$ is torsion-free divisible, since the class of torsion-free divisible groups is closed under extensions. Since $M \in J, T^{\beta}\left(M_{0}\right)=M_{0}$ for some $\beta$ and so $M_{0}$ is torsion-free divisible. This completes the proof.

RemarKs. (1) From the previous lemma and the primary decomposition for torsion abelian groups, the primary decomposition holds for any ring which has no $Q$-type simple modules. In fact, over any ring, if $M \in J$ and $M_{0}=0$, we get $M=\sum_{i=1}^{\infty} M_{i}$.

(2) For any module $M, \sum_{i \neq j} M_{i}$ cannot contain a simple from the class $S_{j}$ and consequently $\sum_{i=0}^{\infty} M_{i}$ is always a direct sum.

LEMma 2.2. The primary decomposition holds for the ring $R$ if and only if $\operatorname{Ext}(S, T)=0$ for each $Q$-type simple $S$ and each module $T \in J$ with $T_{0}=0$.

Proof. The necessity of the condition is clear since if $0 \rightarrow T \rightarrow X \rightarrow S \rightarrow 0$ is exact and the primary decomposition holds, we 
must have $X=X_{0} \oplus \sum_{i=1}^{\infty} X_{i}$ with $X_{0} \approx S$ and this implies that the sequence splits.

To prove that the condition is sufficient, let $M \in J$. We will prove that $M=\sum_{i=1}^{\infty} M_{i}$ by showing that $M / \sum_{i=0}^{\infty} M_{i}$ has no simple submodule. By Lemma 2.1, $\sum_{i=1}^{\infty} M_{i}=M_{t}$, so $\sum_{i=1}^{\infty} M_{i}=M_{0}+M_{t}$. Also by $2.1, M_{0}$ is divisible and so as groups we have

$$
\frac{M}{M_{t}} \approx \frac{M_{0}+M_{t}}{M_{t}} \oplus \frac{K}{M_{t}} .
$$

Thus any subgroup of $M / M_{0}+M_{t}$ is isomorphic to a subgroup of $K / M_{t}$. Hence $M / M_{0}+M_{t}$ contains no $p$-type simple since $K / M_{t}$ is torsion-free as a group.

Assume $M / M_{0}+M_{t}$ has a $Q$-type simple, say $S=X / M_{0}+M_{t}$, $X \subseteq M$. Now $M_{0}+M_{t} / M_{0} \approx M_{t}$ so we have an exact sequence

$$
0 \rightarrow \frac{M_{0}+M_{t}}{M_{0}} \approx M_{t} \rightarrow \frac{X}{M_{0}} \rightarrow \frac{X}{M_{0}+M_{t}}=S \rightarrow 0 .
$$

By hypothesis, this sequence must split, so $X / M_{0}$ contains a $Q$-type simple. Thus $M / M_{0}$ contains a $Q$-type simple and this is a contradiction. Hence $M / \sum_{i=0}^{\infty} M_{i}$ has no simple submodule, so $M=\sum_{i=0}^{\infty} M_{i}$ and the proof is complete.

THEOREM 2.3. The primary decomposition holds for the ring $R$ if and only if

(1) $\left(\prod_{s \in \mathfrak{e}} S / \sum_{s \in \mathfrak{e}} S\right)_{0}=0$, where $\mathfrak{C}$ is a representative set of simples of type $p$.

(2) If $0 \rightarrow P \rightarrow K \rightarrow U \rightarrow 0$ is an exact sequence of $R$-modules with $K$ cyclic, $P \in J_{i}$ for some $i \geqq 1$ and $U$ a $Q$-type simple, then $P$ has nonlimit ordinal T-length.

Proof. To see that the first condition is necessary, suppose $U$ is a $Q$-type simple contained in the factor module $\prod S / \sum S$. Then we have an exact sequence $0 \rightarrow \sum S \rightarrow X \rightarrow U \rightarrow 0$, where $X \subseteq \prod S$. Since the primary decomposition holds, $X \approx U \oplus \sum S$. But then $U \subseteq X$ $\subseteq \prod S$ so

$$
0 \neq \operatorname{Hom}(U, U) \subseteq \operatorname{Hom}\left(U, \prod S\right)=\prod \operatorname{Hom}(U, S)=0
$$

and we have a contradiction.

If $0 \rightarrow P \rightarrow K \rightarrow U \rightarrow 0$ is exact as in (2) above, then $K \approx P \oplus U$ and so $P$ is cyclic. It follows that $P$ has nonlimit ordinal $T$-length.

To show that (1) and (2) are sufficient, we use Lemma 2.2 and show $\operatorname{Ext}(T, U)=0$ for $U$ a $Q$-type simple and $T \in J, T_{0}=0$. 
By previous remarks, $T=\sum_{i=0}^{\infty} T_{i}$ and applying $\operatorname{Hom}(U,-)$ to the exact sequence

$$
0 \rightarrow \sum T_{i} \rightarrow \prod T_{i} \rightarrow \prod T_{i} / \sum T_{i} \rightarrow 0
$$

we get the exact sequence

$$
\operatorname{Hom}\left(U, \Pi T_{i} / \sum T_{i}\right) \rightarrow \operatorname{Ext}\left(U, \sum T_{i}\right) \rightarrow \operatorname{Ext}\left(U, \Pi T_{i}\right) .
$$

But $\operatorname{Ext}\left(U, \prod T_{i}\right)=\prod \operatorname{Ext}\left(U, T_{i}\right)$ so to show that the condition of Lemma 2.2 holds it is sufficient to prove:

(a) $\operatorname{Hom}\left(U, \prod T_{i} / \sum T_{i}\right)=0$,

(b) $\operatorname{Ext}\left(U, T_{i}\right)=0$ for $i \geqq 1$.

By (1) and an easy modification of Lemma 2.2 of [2], (a) holds. We prove that (b) holds by showing $\operatorname{Ext}(U, A)=0$ for any $p_{i}$-primary module $A$. The proof is by induction on the $T$-length of $A$.

If $A$ is a $p_{i}$-primary module of $T$-length one, then $A=\sum S_{\alpha}$ where each $S_{\alpha}$ is a $p_{i}$-type simple. As before

$$
\operatorname{Hom}\left(U, \prod S_{\alpha} / \sum S_{\alpha}\right) \rightarrow \operatorname{Ext}\left(U, \sum S_{\alpha}\right) \rightarrow \operatorname{Ext}\left(U, \prod S_{\alpha}\right)
$$

is exact with right end zero since $\operatorname{Ext}\left(U, S_{\alpha}\right)=0$ because $U$ and $S_{\alpha}$ are simples of different type. Since $p_{i} U=U$, but $p_{i}\left(\prod S_{\alpha} / \sum S_{\alpha}\right)=0$, we must have $\operatorname{Hom}\left(U, \prod S_{\alpha} / \sum S_{\alpha}\right)=0$. Hence $\operatorname{Ext}(U, A)=0$ if $A$ has $T$-length one.

Now assume $\operatorname{Ext}(U, A)=0$ for all $p_{i}$-primary modules $A$ of $T$ length $\alpha<\beta$ and let $B$ have $T$-length $\beta$. If

$$
0 \rightarrow B \rightarrow X \rightarrow U \rightarrow 0
$$

is exact with $B \rightarrow X$ the inclusion map, choose $x \in X-B$. Then

$$
0 \rightarrow B \cap R x \rightarrow R x \rightarrow U \rightarrow 0
$$

is exact, the $T$-length of $B \cap R x$ is less than or equal to $\beta$ and it is not a limit ordinal by (2). Let the $T$-length of $B \cap R x$ be $\alpha+1$. Then

$$
0 \rightarrow \frac{B \cap R x}{T^{\alpha}(B \cap R x)} \rightarrow \frac{R x}{T^{\alpha}(B \cap R x)} \rightarrow U \rightarrow 0
$$

is exact and since $B \cap R x / T^{\alpha}(B \cap R x)$ has $T$-length one, the sequence must split. Thus there is a submodule $K$ of $R x$ containing $T^{\alpha}(B \cap R x)$ with $U \approx K / T^{\alpha}(B \cap R x)$. Then

$$
0 \rightarrow T^{\alpha}(B \cap R x) \rightarrow K \rightarrow U \rightarrow 0
$$

is exact and since $T^{\alpha}(B \cap R x)$ has $T$-length $\alpha<\beta$, this sequence must split. Thus $K$ contains a submodule isomorphic to $U$ and so since 
$K \subseteq R x \subseteq X, X$ has a submodule isomorphic to $U$. It follows that the sequence $\left(^{*}\right)$. splits and this completes the proof.

\section{Applications and examples.}

THEOREM 3.1. Let $R$ be a ring with the property that every maximal left ideal $L$ of $R$, with $R / L$ a Q-type simple, is finitely generated. Then the primary decomposition holds for $R$.

Proof. We apply Theorem 2.3 and show that conditions (1) and (2) hold.

Let $0 \rightarrow P \rightarrow K \rightarrow U \rightarrow 0$ be exact with $K$ cyclic and $U$ a $Q$-type simple. Then for some left ideals $L \subseteq M$ of $R$ we have $P \approx M / L$ and $U \approx R / M$. But then $M$ is finitely generated so $M / L$, and hence $P$, cannot have limit ordinal $T$-length. Thus (2) holds.

Let $U=R\left(x_{s}+\sum S\right) \subseteq \prod S / \sum S$ where the product and sum are taken over the set $\mathfrak{e}$ as in (1) of 2.3. Let $M=\left(\sum S:\left(x_{s}\right)\right)$. Then $U \approx R / M$, so $M=R m_{1}+\cdots+R m_{n}$ is finitely generated, since $U$ is of type $Q$. Now for each $i, m_{i} x_{s}=0$ for all but finitely many $S \in \mathcal{C}$. Hence there is an $x_{s_{0}} \neq 0$ such that $M x_{s_{0}}=0$. But then $R x_{s_{0}} \approx S_{0}$ $\approx R / M \approx U$ and this is a contradiction since $S_{0}$ is of type $p$. Hence (1) of 2.3 is satisfied and the proof is complete. [5].

The following corollary generalizes part of Fuchs' Theorem 72.2

COROLlary 3.2. Let $R$ satisfy the hypothesis of 3.1 and assume nonzero $R$-modules have nonzero socles. Then $R$ is the ring direct sum of two sided ideals $R_{0}, R_{1}, \cdots, R_{n}$ where $R_{0}^{+}$is a direct sum of copies of $Q$ and each $R_{i}^{+}, 1 \leqq i \leqq n$, is a bounded primary group.

Proof. Since nonzero modules have nonzero socles, $R \in J$ and since by 3.1 the primary decomposition holds, we have $R=\sum_{i=0}^{\infty} R_{i}$. Since $R$ has a unit element, $R=R_{0} \oplus \cdots \oplus R_{n}$. Each of the classes $J_{i}$ is closed under homomorphic images and right multiplication by elements of $R$ is a left $R$-homomorphism so each $R_{i}$ is a two-sided ideal. $R_{0}$ is a torsion-free divisible group and so it is a direct sum of copies of $Q$. Each $R_{i}, 1 \leqq i \leqq n$, is a primary group by Lemma 2.1 and since each $R_{i}$ is a ring with unit, it must be a bounded group. This proves the corollary.

To construct examples of non-Artinian rings satisfying the hypotheses of Corollary 3.2, let $P$ be an infinite product of copies of $Z_{p}$. Define the ring $R$ by $R^{+}=P \oplus Z_{p}$ and $\left(p_{1}, i_{1}\right)\left(p_{2}, i_{2}\right)=\left(i_{2} p_{1}+i_{1} p_{2}, i_{1} i_{2}\right)$. Then $P$ is the socle of $R$ and $R / P \approx Z_{p}$; so nonzero modules have nonzero socles. Since $R$ has no $Q$-type simples, the hypothesis of 4.1 is clearly satisfied. Since every subgroup of $P$ is an ideal of $R$, it is clear that $R$ is neither Artinian or Noetherian. 


\section{REFERENCES}

1. J. S. Alin, Primary decomposition of modules, Math. Z. 107 (1968), 319-325. MR 39 \#258.

2. S. E. Dickson, Decomposition of modules. I. Classical rings, Math. Z. 90 (1965), 9-13. MR 32 \#2445.

3. - Decomposition of modules. II. Rings without chain conditions, Math. Z. 104 (1968), 349-357. MR 37 \#5252.

4. - A torsion theory for Abelian categories, Trans. Amer. Math. Soc. 121 (1966), 223-235. MR 33 \#162.

5. L. Fuchs, Abelian groups, Akad. Kiad6, Budapest, 1958; republished by Internat. Series of Monographs on Pure and Appl. Math., Pergamon Press, New York, 1960. MR $21 \# 5672$; MR $22 \# 2644$.

6. S. Mac Lane, Homology, Die Grundlehren der math. Wissenschaften, Band 114, Academic Press, New York; Springer-Verlag, Berlin, 1963. MR 28 \#122.

University of Utah, Salt Lake City, Utah 84112 